\title{
Neck Dissection and Thoracoscopic Esophagectomy in Esophageal Cancer with Aberrant Subclavian Artery
}

\author{
HIROYUKI KITAGAWA, TSUTOMU NAMIKAWA and KAZUHIRO HANAZAKI
}

Department of Surgery, Kochi Medical School, Nankoku, Japan

\begin{abstract}
We report a case of esophageal cancer with a non-recurrent inferior laryngeal nerve associated with aberrant right subclavian artery treated with neck dissection followed by thoracoscopic esophagectomy. A 60-year-old man experienced esophageal cancer, hoarseness, and left supraclavicular lymph node swelling was noted on endoscopy. Computed tomography revealed an aberrant right subclavian artery between the esophagus and vertebrae. We administered neo-adjuvant chemotherapy and performed thoracoscopic esophagectomy. During the neck dissection, we confirmed a non-recurrent inferior laryngeal nerve along the inferior thyroid artery. After the neck dissection, we performed thoracoscopic esophagectomy. We confirmed an aberrant right subclavian artery arising from the aortic arch, and resected the left recurrent nerve due to cancer invasion. No postoperative complication was observed, and the patient was discharged 17 days after surgery. Thus, we recommend prior neck dissection in cases involving aberrant right subclavian artery during esophagectomy.
\end{abstract}

In esophageal squamous cell carcinoma (ESCC), the dissection of the upper mediastinal lymph node including around the recurrent laryngeal nerve (RLN) is important during esophagectomy due to the high incidence of metastasis (1).

However, an aberrant right subclavian artery (ARSA) is a vessel anomaly reported in about $0.6-1.3 \%$ of patients (2), usually with a non-recurrent inferior laryngeal nerve (NRILN). Herein, we report a case of a patient with ESCC with a NRILN associated with an ARSA treated with prior neck dissection followed by thoracoscopic esophagectomy (TSE).

Correspondence to: Tsutomu Namikawa, Department of Surgery, Kochi Medical School, Kohasu-Okocho, Nankoku, Kochi, 7838505, Japan. Tel: +81 888802370, Fax: +81 888802371, e-mail: tsutomun@kochi-u.ac.jp

Key Words: Aberrant right subclavian artery, esophageal cancer, nonrecurrent inferior laryngeal nerve, thoracoscopic esophagectomy.

\section{Case Report}

A 60-year-old man experienced left supraclavicular lymph node swelling. Endoscopy revealed a circumference type-2 tumor, located 25 to $32 \mathrm{~cm}$ from the teeth. Biopsy revealed a squamous cell carcinoma. Computed tomography (CT) revealed an upper thoracic esophageal tumor with bilateral supraclavicular lymph node and mediastinal lymph node metastasis (Figure 1). Threedimensional CT also demonstrated an ARSA as the fourth branch of the aortic arch (Figure 2). A positron emission tomography CT showed no distant metastasis to the bone, lungs, or liver. We diagnosed the tumor as an advanced upper thoracic ESCC, and administered neo-adjuvant chemotherapy with docetaxel, cisplatin, and fluorouracil, followed by TSE. We obtained informed consent from the patient.

After completing the neo-adjuvant chemotherapy, reduction of the tumor, but no change of the lymph node metastasis was seen on CT. The patient's hoarseness and esophageal stenosis worsened, and he was unable to eat any food. We decided to perform a prior neck dissection followed by TSE to confirm the NRILN because the left recurrent nerve may have been invaded with cancer and require a resection during the lymph node dissection.

During the neck dissection, we confirmed the NRILN along the inferior thyroid artery. The left supraclavicular lymph node had a cancerous invasion that extended to the right jugular vein and right thoracic duct; therefore, we resected them. After neck dissection, we performed TSE in the prone position. We confirmed that the ARSA arose from the aortic arch (Figure 3). The azygos vein, vagal nerve, and the left recurrent nerve had cancerous invasions; thus, we also resected them. After TSE, we created a gastric tube and post-sternum route reconstruction with a circular stapler anastomosis was made at the neck.

No postoperative complications were observed, and the patient was discharged 17 days after surgery.

\section{Discussion}

An ARSA can be identified by CT (3). When CT reveals an ARSA, the existence of the NRILN should be considered. 


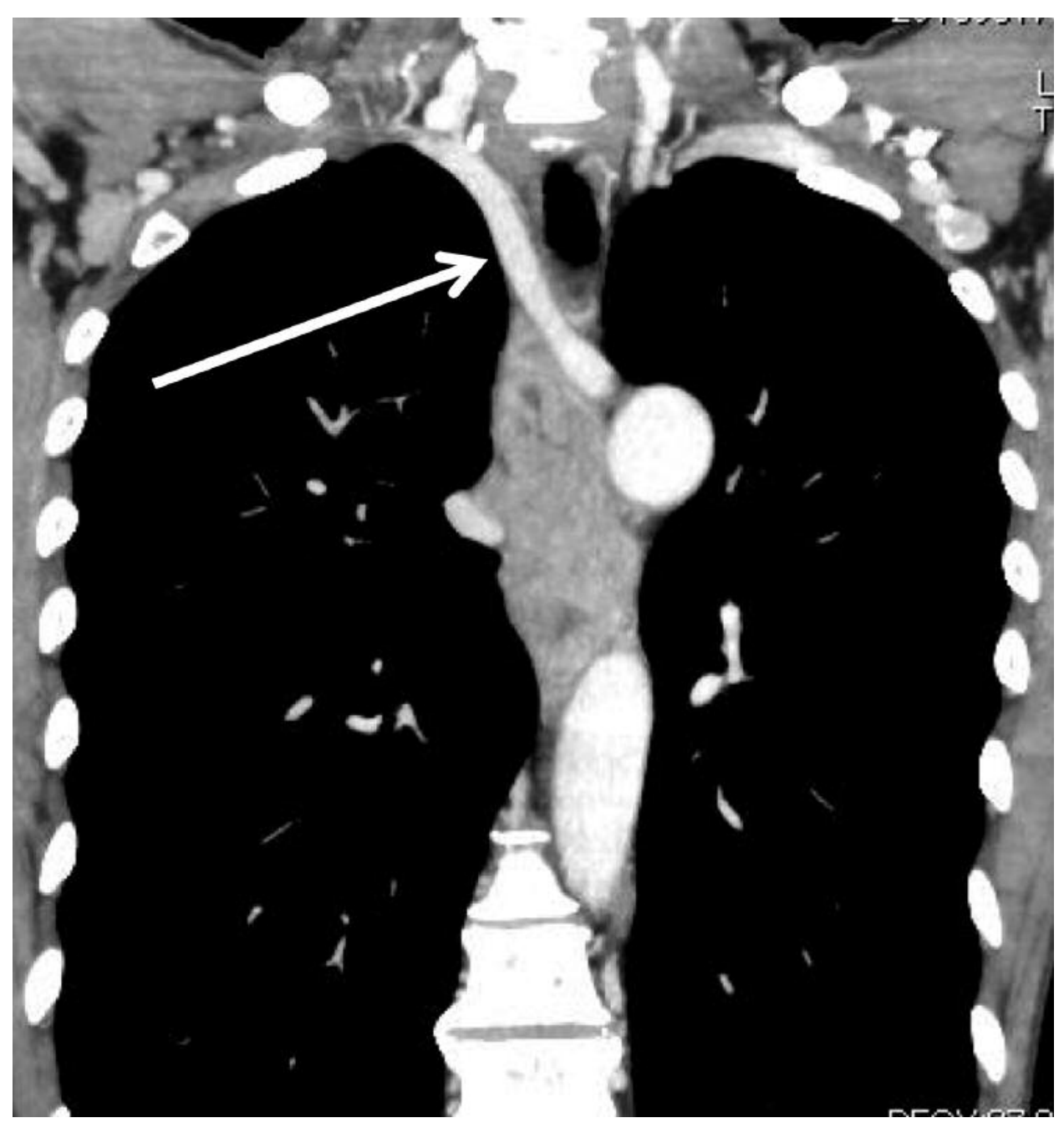

Figure 1. Computed tomography showed an upper thoracic esophageal tumor and aberrant right subclavian artery (arrow).

Toniato et al. classified the pattern of NRILN into the following three types: type 1 , arising from the vagal nerve and running directly into the larynx; type $2 \mathrm{a}$, running together with the inferior thyroid artery; type $2 b$, running under the inferior thyroid artery (4). Our patient was classified as having type 2a, and we detected the NRIRN during the neck dissection. This led to the decision to resect the left RLN invaded by cancer.

We recommend prior neck dissection before thoracic esophagectomy for the following reasons. Firstly, the space between the ARSA and trachea is narrow compared to that in the normal individual, and lymph node dissection around the left RLN in the thorax is difficult. Secondly, the decision as to whether to resect or preserve the left RLN if it is invaded by cancer can be after confirming the right NRILN during the prior neck dissection. Thirdly, patients with ARSA might have a right thoracic duct (5). In order to avoid postoperative chylothorax, attention should be paid to the right thoracic duct during the neck dissection.

\section{Conclusion}

We recommend neck dissection prior to TSE in cases of thoracic ESCC with ARSA and NRILN.

\section{Conflicts of Interest}

None declared.

\section{Funding}

No funding was received on behalf of this research. 


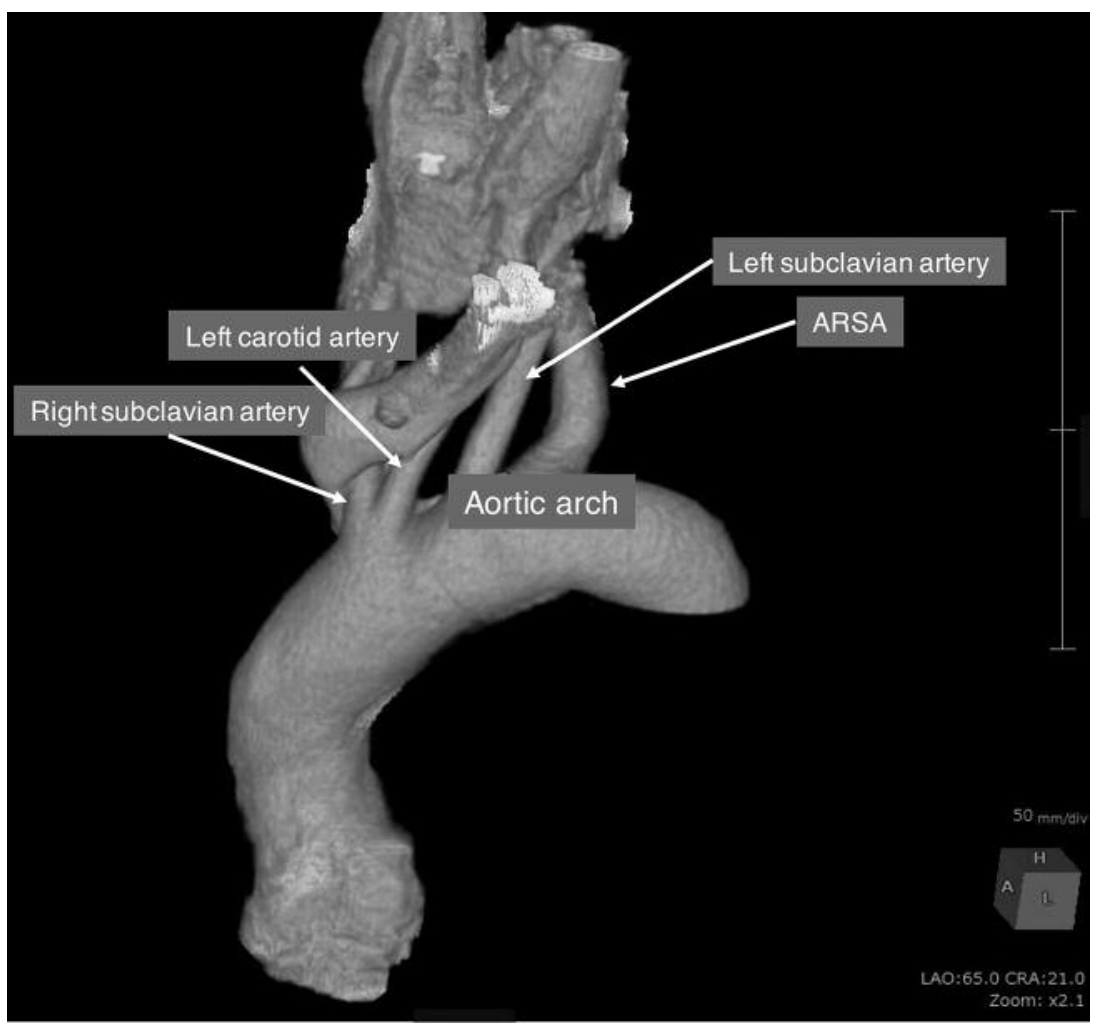

Figure 2. 3-D computed tomography showed an aberrant right subclavian artery arising from the fourth branch of the aortic arch.

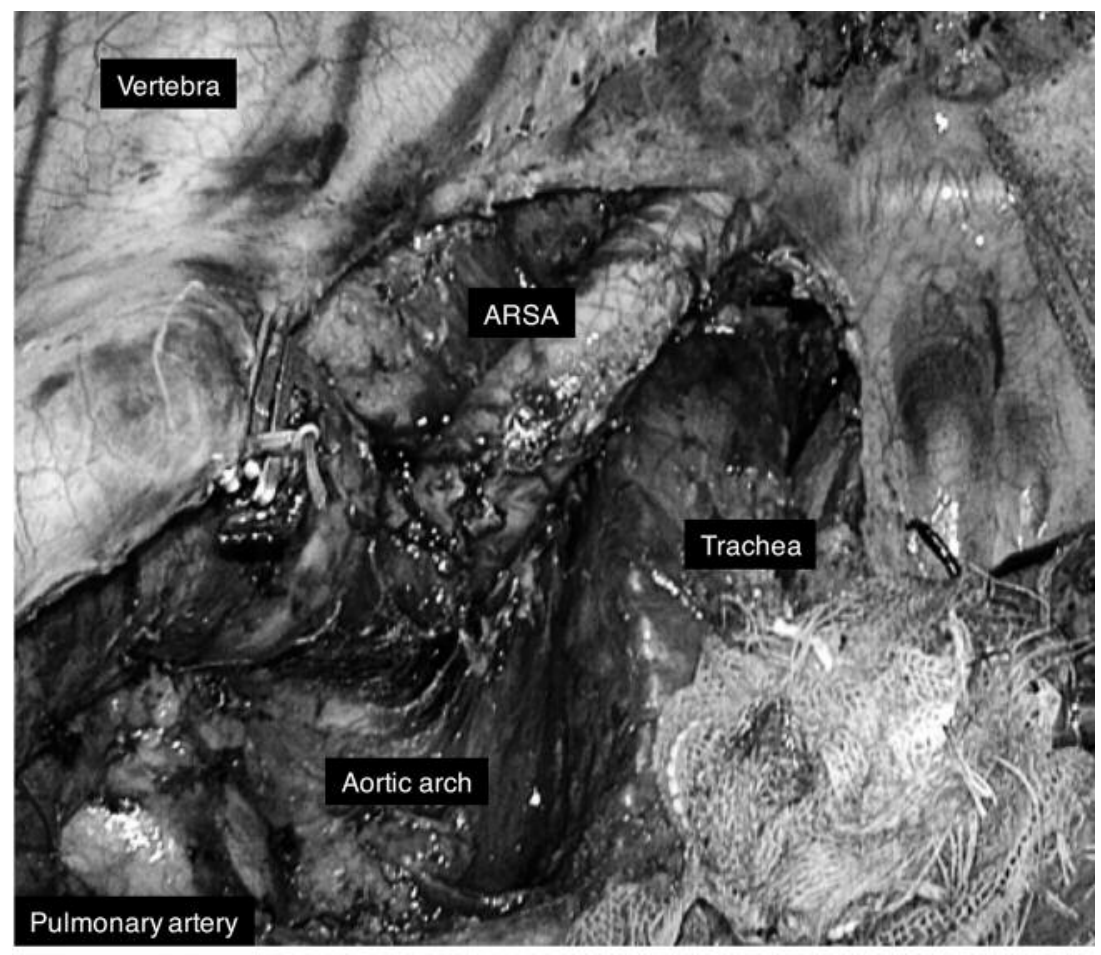

Figure 3. Intrathoracic view during the thoracoscopic esophagectomy. The aberrant right subclavian artery (ARSA) was found to arise from the aortic arch and run through dorsal side of the trachea. 


\section{References}

1 Tachimori Y, Nagai Y, Kanamori N, Hokamura N and Igaki H: Pattern of lymph node metastases of esophageal squamous cell carcinoma based on the anatomical lymphatic drainage system. Dis Esophagus 24: 33-38, 2011.

2 Henry JF, Audiffret J, Denizot A and Plan M: The nonrecurrent inferior laryngeal nerve: review of 33 cases, including two on the left side. Surgery 104: 977-984, 1988.

3 Watanabe A, Kawabori S, Osanai H, Taniguchi $M$ and Hosokawa M: Preoperative computed tomography diagnosis of non-recurrent inferior laryngeal nerve. Laryngoscope 111: 17561759, 2001.
4 Toniato A, Mazzarotto R, Piotto A, Bernante P, Pagetta C and Pelizzo MR: Identification of the nonrecurrent laryngeal nerve during thyroid surgery: 20-year experience. World J Surg 28: 659-661, 2004.

5 Oya $S$, Mine S, Watanabe $M$ and Yamada K: Oesophageal cancer with an aberrant right subclavian artery accompanied by a thoracic duct anomaly. Eur J Cardiothorac Surg 48: e55-57, 2015.

Received May 18, 2017

Revised June 2, 2017

Accepted June 6, 2017 